\title{
Im Teamwork zum Therapieerfolg
}

Der Einsatz des selektiven Immunsuppresivums Belimumab (Benlysta ${ }^{\oplus}$ ) bietet eine erfolgversprechende Therapieoption beim systemischen Lupus erythematodes [1, 2]. Je besser die Kooperation zwischen Arzt und Patient, desto höher die Erfolgsrate.

"Der Lupus erythematodes ist eine Krankheit, die sehr unterschiedlich verlaufen und verschiedene Organsysteme befallen kann und entsprechend vielfältige Leiden verursacht", erläutert Univ.Prof. Dr. Erika Aberer von der MedUni Graz. „Als Folge davon ist bei den meisten Patienten die Lebensqualität massiv beeinträchtigt."

\section{„An amazing journey“ [3]}

Wie massiv die Krankheit das Leben einer Patientin prägt, zeigt $O A ̈$ Dr. Ulrike Stuby, von der 2. Med. Abteilung am AKH Linz anhand des Falles einer heute 40-jährigen Musikerin, bei der sich vor rund 20 Jahren die Krankheit erstmals zeigte. Die ersten Symptome waren schmerzhafte, geschwollene Fingergelenke. Anamnese und klinische Tests ergaben, dass es sich um SLE handelte. Die Therapie mit Glukokortikoiden, Chloroquinphosphat, Tetracosactid-hexaacetat und NSAR (bei Bedarf) zeigte keine nachhaltige Wirkung. Die Patientin litt unter immer wiederkehrenden Krankheitsschüben, die ihr Studium am Salzburger Mozarteum und später ihre berufliche Tätigkeit massiv erschwerten: So traten in den Folgejahren (teils vorübergehend) u.a. Allopezie, Exantheme und Extrasystolen verbunden mit einer Pleuritis auf. Typisch waren „Ruhephasen“ der Erkrankung, auf die plötzliche Schübe folgten. Während einer Urlaubsreise traten Kopfschmerzen, Sehstörungen und Bewusstseinsverlust auf - Symptome, die auf eine Beteiligung des ZNS (zerebrale Vaskulitis) hindeuteten und nur mit hohen Kortisondosen in den Griff zu bekommen waren. Weiters war eine Antikoagulationstherapie und eine zeitweilige Apherese erforderlich. "Diese Situation war äußerst kritisch, auch weil sich bei der Patientin trotz
Kortisongabe ein erheblicher Gewichtsverlust einstellte", sagt Stuby. Die Lebensqualität der Patientin war massiv beeinträchtigt, zeitweise konnte sie ohne fremde Hilfe kaum mehr das Bett verlassen. Entsprechend musste sie ihre beruflichen Ambitionen zurückschrauben; sie war im Dauerkrankenstand und musste schließlich ihren Beruf ganz aufgeben.

In den folgenden Jahren kam es zu Osteopenie, Thrombopenie, Polyarthritis, Fatigue, Fieberschüben, Plegie - die jeweils medikamentös behandelt wurden. "Typisch war, dass meist zu Beginn der Therapie eine rasche Besserung der Beschwerden eintrat, die aber nur von kurzer Dauer war", erklärt Stuby. Infolge der erheblichen körperlichen Belastung stellte sich bei der Patientin - fast zwangsläufig eine Depression ein.

\section{Einsatz von Belimumab}

Nachdem die Patientin schon vorher mehrere Therapiezyklen mit einem monoklonalen Antikörper durchlaufen hatte (bei dem allerdings ein früher klinischer Wirkverlust eingetreten war), wurde 2011 eine Therapie mit Belimumab eingeleitet. Belimumab ist ein humaner monoklonaler IgG1 $\lambda$-Antikörper, der die Bindung von löslichem BLyS, einem B-Zell-Überlebensfaktor, an seinen Rezeptor auf den B-Zellen blockiert. Belimumab bindet nicht direkt an B-Zellen, sondern hemmt durch Bindung an BLyS das Überleben dieser Zellen, einschließlich der autoreaktiven BZellen, und reduziert die Ausdifferenzierung von B-Zellen zu Immunglobulin-bildenden Plasmazellen. [4] Die Substanz wird als Infusion verabreicht [4], was bei der Patientin zwar Übelkeit auslöste, nichtsdestoweniger aber von inr selbst als "gut verträglich" bezeichnet wurde. Außerdem sprach sie auf die neue Therapiestrategie gut an (Standard: Belimumab, Cotherapie mit Hydroxychloroquinsulfat, Phenprocoumon sowie Valacyclovir als Dauerprophylaxe wegen der durch die Immunsuppression bestehenden Gefahr von Herpesinfektionen). Im ersten Jahr der Behandlung litt die Patientin häufiger unter Infekten, deren Auftreten jedoch im weiteren Verlauf zurückging. Auch Gewichtsschwankungen und Schwankungen der Blutgerinnungsparameter konnten durch eine Dosisanpassung von Belimumab in den Griff bekommen werden.

„Positiv ist zu vermerken, dass es mit dieser Therapie möglich wurde, die Kortisongabe kontinuierlich zu reduzieren", erklärt Stuby. Neben der Besserung der klinischen Werte ist vor allem die von der Patientin subjektiv konstatierte allgemeine Konsolidierung ihres Zustandes von großer Bedeutung: Sie konnte ihr Masterstudium doch noch abschließen. Mittlerweile gibt sie sogar wieder Konzerte.

Mag. Ingo Schlager

\section{Referenzen:}

1. Navarra SV et al. „Efficacy and safety of belimumab in patients with active systemic lupus erythematosus: a randomised, placebo-controlled, phase 3 trial", in: Lancet 2011 Feb 26;377(9767):721 731.

2. Furie R. et al. „A phase III, randomized placebo-controlled study of belimumab, a monoclonal antibody that inhibits B lymphocyte stimulator, in patients with systemic lupus erythematosus", in: Arthrits Rheum. 2011 Dec;63(12):3918-30.

3. "An amazing journey“, Vortrag von $\mathrm{OA}$ Dr. Ulrike Stuby im Rahmen des Satellitensymposiums ,...aber nicht nur Rheumatologen sehen Lupus-Patienten“ der GlaxoSmithKline Pharma GmbH bei der Jahrestagung der ÖGR vom 28. bis 30. November 2013 in Wien.

4. Fachinformation Benlysta 